\title{
Analysis of the Trypanosome Flagellar Proteome Using a Combined Electron Transfer/Collisionally Activated Dissociation Strategy
}

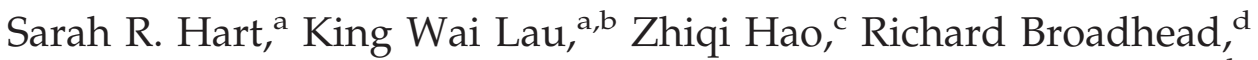

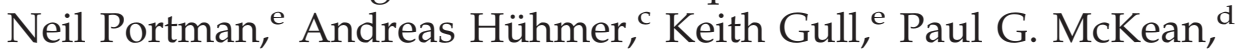 \\ Simon J. Hubbard, ${ }^{\mathrm{b}}$ and Simon J. Gaskell ${ }^{\mathrm{a}}$ \\ ${ }^{a}$ Michael Barber Centre for Mass Spectrometry, School of Chemistry and Manchester Interdisciplinary \\ Biocentre, University of Manchester, Manchester, United Kingdom \\ ${ }^{b}$ Faculty of Life Sciences, University of Manchester, Manchester, United Kingdom \\ c Thermo Fisher Scientific, San Jose, California, USA \\ ${ }^{\mathrm{d}}$ Department of Biological Sciences, Lancaster University, Lancaster, United Kingdom \\ e Sir William Dunn School of Pathology, University of Oxford, Oxford, United Kingdom
}

The use of electron-transfer dissociation as an alternative peptide ion activation method for generation of protein sequence information is examined here in comparison with the conventional method of choice, collisionally activated dissociation, using a linear ion trapping instrument. Direct comparability between collisionally and electron-transfer-activated product ion data were ensured by employing an activation-switching method during acquisition, sequentially activating precisely the same precursor ion species with each fragmentation method in turn. Sequest (Thermo Fisher Scientific, San Jose, CA) searching of product ion data generated an overlapping yet distinct pool of polypeptide identifications from the products of collisional and electron-transfer-mediated activation products. To provide a highly confident set of protein recognitions, identification data were filtered using parameters that achieved a peptide false discovery rate of $1 \%$, with two or more independent peptide assignments required for each protein. The use of electron transfer dissociation (ETD) has allowed us to identify additional peptides where the quality of product ion data generated by collisionally activated dissociation (CAD) was insufficient to infer peptide sequence. Thus, a combined ETD/CAD approach leads to the recognition of more peptides and proteins than are achieved using peptide analysis by CAD- or ETD-based tandem mass spectrometry alone. (J Am Soc Mass Spectrom 2009, 20, 167-175) @ 2009 Published by Elsevier Inc. on behalf of American Society for Mass Spectrometry

$\mathrm{T}$ The field of protein sequencing and proteome characterization has been revolutionized by the application of tandem mass spectrometry to sequence analysis of proteolytic protein fragments. Rapid, sensitive analysis of multiple peptides in an automated fashion, and interrogation of protein sequence databases with the fragment ion data thus generated, have together enabled a wide variety of proteomic studies, providing a vast array of new biochemical targets for functional validation.

Whilst the popular analysis of peptides via collisionally activated dissociation (CAD) is rapid, and results in reproducible and somewhat predictable fragmentation behavior for a given peptide sequence, a substantial proportion of peptide product ion mass spectra do not

Address reprint requests to Dr. S. J. Gaskell, University of Manchester, Manchester Interdisciplinary Biocentre, John Garside Building, 131 Princess Street, Manchester, M1 7DN, United Kingdom. E-mail: simon.gaskell@manchester.ac.uk result in successful sequence identification [1-3]. In part, this results from the co-analysis of multiple components of close mass-to-charge (for example, those co-eluting from an online chromatographic column); these may correspond to unrelated peptides or nonpeptidic structurally unrelated compounds. More significantly, in other cases, difficulties arise from the failure of peptide precursor ions to yield fragmentation informative of primary sequence. For example, the predominance of a single or few fragmentation pathways (as may be observed with post-translationally modified structures) may preclude the direct derivation of significant sequence information [4]. Furthermore, CAD (and other ion heating methods such as infra-red multiphoton dissociation) necessarily becomes less effective as the number of vibrational degrees of freedom in the precursor ion increases. In practice, doubly protonated peptides arising from peptides of up to $\sim 25$ residues typically yield useful information, although examples
(C) 2009 Published by Elsevier Inc. on behalf of American Society for Mass Spectrometry. $1044-0305 / 09 / \$ 32.00$

doi:10.1016/j.jasms.2008.08.014
Published online September 3, 2008 Received January 16, 2008 Revised August 14, 2008 Accepted August 14, 2008 
exist of successful analyses of larger, even intact protein analytes $[5,6,7]$. Whilst the quantitative significance of this limitation to proteome analysis is not yet well understood, there is clear benefit to methods that extend the analytical capability to investigate larger polypeptides.

The use of alternative ion activation methods may help overcome these limitations in tandem mass spectrometrybased proteomics. Electron capture dissociation (ECD), for example, has been shown to achieve effective cleavage of large polypeptides and small proteins [8]. This method induces peptide fragmentation, primarily at $\mathrm{N}-\mathrm{C} \alpha$ bonds, using low-energy electrons [9]. Discussion continues as to the precise nature of the fragmentation mechanisms induced by ECD $[10,11,12]$, but clearly the radical-induced fragmentations occur at low internal energy and are qualitatively different to those induced by CAD [13]. For instance, facile neutral loss of phosphoric acid, as commonly observed in CAD spectra of phosphopeptides, is rarely observed using ECD. This has prompted a strong focus on the use of ECD for post-translational modification analysis [14], but ECD has also proven successful more generally in generating peptide and protein identification data $[8,9,13,15,16]$. The implementation of ECD is however largely restricted to ion cyclotron resonance instruments. Furthermore, the efficiency of the process is limited by the cross-section of interaction between the trapped ions and the electron beam. Thus, it is attractive to achieve electron-transfer to (multiply charged) precursor ions via the use of intermediary anionic reactant species. This method, termed electron-transfer dissociation (ETD), has been implemented upon both linear and quadrupole ion traps $[17,18]$, and yields product ion populations similar to those generated in ECD. The efficiency of the ETD process may be optimized by adjustment of the precursor ion population and the interaction time with the reagent anion $[17,19]$. A recent article indicating the complementary value of ETD to conventional CAD analysis was published by Good et al. during preparation of this study, and will be discussed in further detail below [20].

In anticipation of the complementary value of CAD and ETD for proteome analysis, we have therefore examined the utility of a combined approach. These investigations concerned the flagellum of the African trypanosome, Trypanosoma brucei. The flagellum is an organelle, that is intimately involved in both the motility and infective capacity of this important human pathogen. In previous studies of the Trypanosoma brucei flagellar proteome $(\mathrm{TbFP})$, we used gel electrophoresis for protein separation and reverse-phase high-performance liquid chromatography (RP-HPLC), coupled to CAD tandem mass spectrometry (MS/MS) of proteolytic peptides using a quadrupoletime of flight instrument. Previously, we identified the expression products of 337 candidate flagellar proteins, following post hoc bioinformatic filtering on the basis of predicted protein isoelectric point [21]. Here, we complement our previous strategy by using ETD/CAD switching during RP-HPLC-MS/MS analysis of peptide fractions from strong cation exchange separation of total flagellum protein digests.

\section{Experimental}

\section{Chemicals and Materials}

Triethyl ammonium bicarbonate, tris(carboxyethyl)phosphine, methyl methanethiosulfonate, HPLC-grade water and acetonitrile, and proteomics-grade trypsin were obtained from Sigma (Poole, Dorset, UK). Sequencinggrade endoproteinase Lys-C was purchased from Roche Molecular Biochemicals (Lewes, East Sussex, UK). Phosphoric acid and potassium dihydrogen phosphate were from Fluka (Poole, Dorset, UK), and potassium chloride was from Riedel de Haan (Poole, Dorset, UK).

\section{Sample Preparation}

Trypanosoma brucei flagellum samples were prepared as described previously [21]. Briefly, doxycyclin-induced snl2 RNAi mutant cultured trypanosome cells were lysed and subjected to flagellar preparation. Pelleted flagellar proteins (approximately $12 \mu \mathrm{g}$ by Bradford assay) were dissolved in $500 \times 10^{-3} \mathrm{M}$ triethyl ammonium bicarbonate solution containing $0.1 \%$ SDS (wt/vol), reduced using $1 \times 10^{-3} \mathrm{M}$ tris(carboxyethyl)phosphine $\left(1 \mathrm{~h}\right.$ at $\left.60^{\circ} \mathrm{C}\right)$ and alkylated using $1 \times 10^{-3} \mathrm{M}$ methyl methanethiosulfonate (10 $\mathrm{min}$ at room-temperature). Proteins were then digested using either trypsin or endoproteinase Lys-C overnight at $37^{\circ} \mathrm{C}$.

\section{Peptide Strong Cation Exchange Chromatography}

Following digestion, peptides were subjected to vacuum centrifugation to remove volatile base. The digest mixtures were then dissolved in strong cation exchange buffer $\mathrm{A}\left(10 \times 10^{-3} \mathrm{M} \mathrm{KH}_{2} \mathrm{PO}_{4}, \mathrm{pH} 2.8,20 \% \mathrm{CH}_{3} \mathrm{CN}\right)$, and acidified using $o$-phosphoric acid. Peptides were loaded onto a polysulfoethyl A column $(200 \times 2.1 \mathrm{~mm}$, $5 \mu \mathrm{m}, 300 \AA$ A, PolyLC; Hichrom, Theale, Berkshire, UK), and eluted using a linear gradient over $40 \mathrm{~min}$ of $0 \%$ to $40 \%$ Solvent B $\left(10 \times 10^{-3} \mathrm{M} \mathrm{KH}_{2} \mathrm{PO}_{4}, 500 \times 10^{-3} \mathrm{M}\right.$ $\left.\mathrm{KCl}, \mathrm{pH} 2.8,20 \% \mathrm{CH}_{3} \mathrm{CN}\right)$, using an Agilent $\mathrm{HP} 1100$ HPLC system (Agilent, Stockport, Cheshire, UK) interfaced with an AB 783 programmable absorbance detector (Applied Biosystems, Framingham, MA). Fractions were collected at 1-min intervals throughout the gradient. Collected fractions were dried in vacuo to remove organic solvent and stored at $-20{ }^{\circ} \mathrm{C}$ before analysis.

\section{Mass Spectrometry}

These experiments were performed using a modified linear ion trap coupled to an additional chemical ionization source at the rear instrument modification port (Finnigan LTQ and Finnigan 4500 CI source; both Thermo Fisher Scientific., San Jose, CA), as described by 
Syka et al. [17]. Electrons were transferred to gas-phase analyte ions via fluoranthene anions generated in the CI source, and ETD ion/ion reaction time was maintained at $100 \mathrm{~ms}$, with 200,000 anions injected for each scan. The instrument was operated using a modified version of Xcalibur software (ThermoFisher Scientific). Peptide fractions were dissolved in $0.1 \%$ formic acid in water (HPLC Solvent A) for mass spectrometric analysis. Peptides (8 $\mu \mathrm{L}$ injection volume) were introduced via an HP1100 HPLC system (Agilent, Palo Alto, CA), with an inline flow splitter reducing the flow rate to $300 \mathrm{~nL} \mathrm{~min}^{-1}$, and separated using a column packed with Reprosil C18-aq $3 \mu \mathrm{m}$ (Dr, Maisch, Ammerbuch-Entringen, Germany), with a C18 trap column of Aqua $5 \mu \mathrm{m}$ particles (Phenomenex, Macclesfield, Cheshire, UK). Peptides were separated using the following gradient conditions: (1) $20 \mathrm{~min}$ at $100 \%$ Solvent A for sample loading, (2) linear gradient to $40 \%$ Solvent B ( $1 \%$ formic acid in $\mathrm{CH}_{3} \mathrm{CN}$ ) over 40 min, (3) increasing to $80 \%$ B over $10 \mathrm{~min}$, and finally (4) constant $80 \%$ B for $10 \mathrm{~min}$. Product ion spectra were sequentially recorded for each selected precursor ion using CAD and ETD to enable direct comparability of the two datasets. For automatic function switching analysis, the three most abundant precursors were selected from each survey (MS) scan ( $m / z$ 400-2000), with dynamic exclusion of previously-selected precursors over a 2 min window. Subsequent product ion scans in CAD and ETD modes were performed sequentially for each chosen precursor.

\section{Data Processing}

Raw mass spectrometric data were used to generate text (.dta) files for further processing via the TurboSequest DTA module of Bioworks (Thermo Fisher Scientific). Product ion spectra generated via CAD and ETD were separated using a custom-written batch file for database searching using appropriate search parameters for each activation method, and ETD data and CAD data were then separately subjected to searching against a concatenated forward/reverse T. brucei genomic database (generated using version 4 release, dated July 2005, available from the Sanger Institute at ftp://ftp.sanger.ac.uk/pub/ databases/T.brucei_sequences/T.brucei_genome_v4/), using Sequest (Thermo Fisher Scientific). For all data, up to two missed cleavages with fully enzymatic products were allowed, assuming all cysteine residues were modified by methyl methanethiosulfonate (45.99 Da), and allowing partial modification of methionine residues by oxidation (15.99 Da). Peptide tolerances of 2 Da were allowed to compensate for mass uncertainty of large, highly charged precursor ions, whilst product ion tolerances were set to $1 \mathrm{Da}$. For CAD data, allowed ion types were $b$ and $y$, whilst for ETD data, ion types were $c$ and $z$. Lists of peptides identified (as .dta and .out files) were interrogated using custom perlscripts to allow determination of false discovery rates (FDR) using $\mathrm{XCorr}$ and $\Delta \mathrm{Cn}$ cutoffs, to permit subsequent filtering using a set FDR, in a modified version of the method detailed by Peng et al. [22]. False discovery rates were estimated from the ratio of false positive (FP) peptide identifications from the reverse (decoy) peptides to true positive (TP) identifications from the forward peptides in the concatenated database. Appropriate values of XCorr and $\Delta \mathrm{Cn}$ yielding specific FDRs were achieved using a grid (XCorr minimum 1 , step size $0.1, \Delta$ Cn minimum 0.08 , step size 0.01; see Supplementary Figure 3, which can be found in the electronic version of this article). The XCorr $/ \Delta C n$ pairing yielding the maximum number of peptide identifications at a set FDR was reported and the attendant peptide identifications considered; in this way a consistent data processing pipeline was used throughout. XCorr measures the extent of similarity between an experimental spectrum and the theoretical spectrum of a candidate sequence, whilst $\Delta \mathrm{Cn}$ measures the degree of difference between the correlation of the top hitting candidate sequence and the next candidate to the experimental data [23].

\section{Results and Discussion}

\section{CAD and ETD Product Ion Spectra}

Figure 1 illustrates typical spectra as observed during an LC-MS/MS analysis of peptides with automated switching between CAD and ETD. ETD of doubly protonated peptide ions (Figure 1a) indicates low apparent decomposition efficiency, with the intact peptide radical cation as the principal product. Minor yields are frequently observed of z-series ions, with a few examples of c-series products typically being observed (one in Figure 1a). As has been previously reported, performing ETD on doubly charged peptide precursor ions typically generates fragment ion spectra with few cleavage products [17-19]. Amelioration of this poor spectral quality may be possible using sequential electron-transfer and collisional warming of doubly protonated precursor ions, via fragmentations associated with ETD rather than CAD [24].

For more highly charged peptide precursor ions (those bearing three or more protons), highly informative ETD product ion spectra were observed (Figure $1 \mathrm{~b}$ and c), featuring numerous c- and z-series ions enabling the elucidation of a high proportion of the peptide primary sequence. Such spectra therefore facilitated automated peptide identification using the Sequest search program. By contrast, CAD spectra generated from triply and quadruply protonated species yielded relatively little sequence information content, and hence did not typically result in high-confidence peptide identifications. Thus, the optimal protonation state of peptide precursors differs significantly between ETD and CAD; this phenomenon has been reported previously in other studies [17, 19, 24], and reflects inherent properties of peptides, the most important of these being the number of basic (Arg, Lys, His) residues. A large number of peptide sequences show preferential highquality fragmentation by either CAD or ETD, as indicated in Figure 1. Product ion spectra show numerous qualitative differences; typically ETD spectra show relative uniformity of ETD product ion intensity, and are less dominated by 


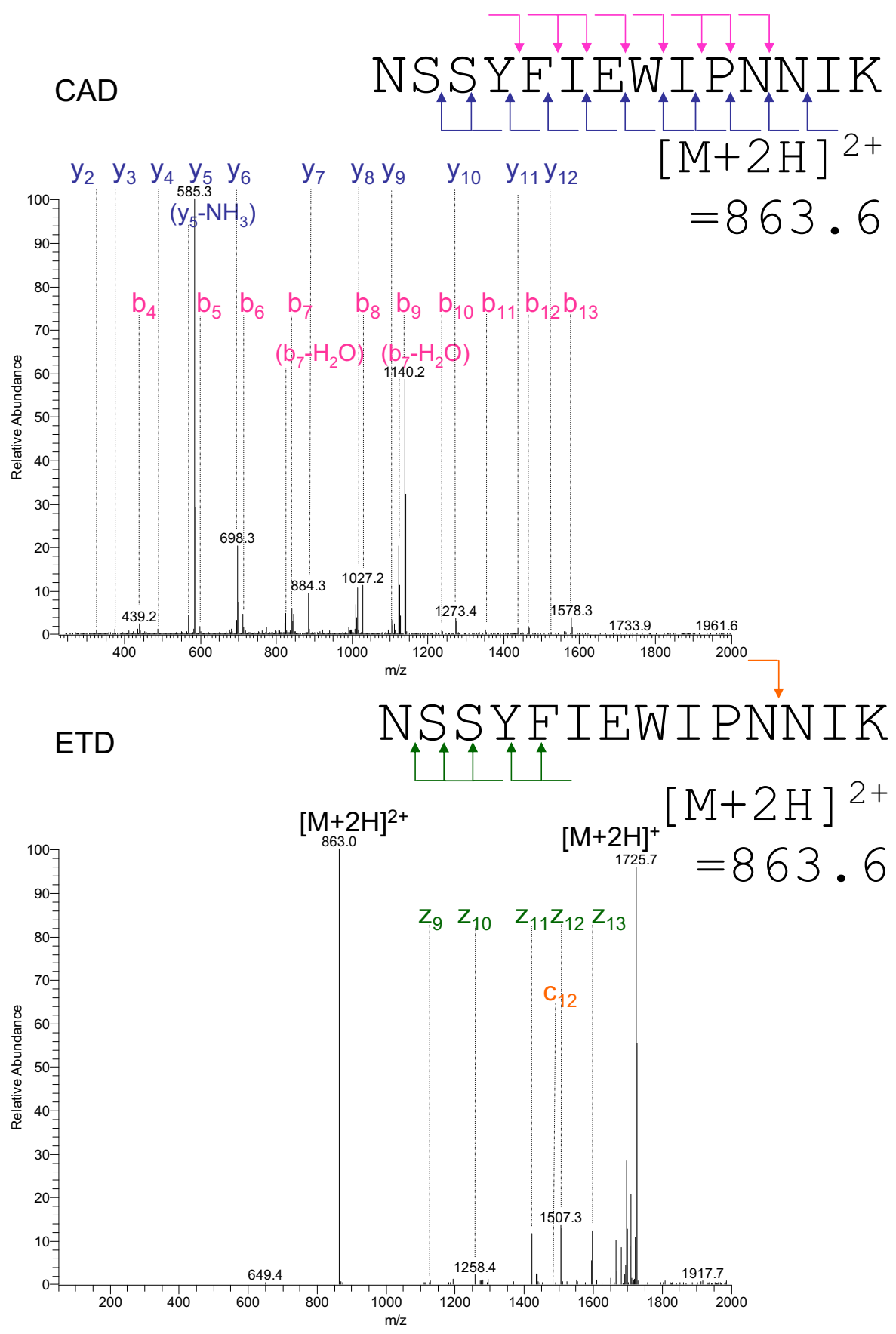

Figure 1. Comparison of spectra generated by CAD and ETD on the same precursor ion types. Peptide ions were generated by ESI and subjected to either CAD (upper panels) or ETD (lower panels). Major identified product ions as labeled upon spectra according to either $\mathrm{b}$ and $\mathrm{y}$ series (CAD) or $\mathrm{c}$ and $\mathrm{z}$ series (ETD) are indicated by arrows upon the N- or C-terminal residue. (a) Product ion spectra generated from a doubly charged peptide precursor of sequence NSSYFIEWIPNNIK, corresponding to a peptide from $T$. brucei $\beta$-tubulin (GeneDB accession Tb927.1.2370, NCBI accession no. XP_846746). Spectroscopic data shown in (b) give complete sequence coverage to a peptide sequence MREIVCVQAGQCGNQIGSK, corresponding to the unmodified N-terminal peptide from T. brucei $\beta$-tubulin (GeneDB accession Tb927.1.2370, NCBI accession no. XP_846746). (c) Example of typical spectra acquired using a quadruply charged precursor generated via enzymolysis with Lys-C, sequence ETLREIISEHDIVDTK, which corresponds to a peptide from a T. brucei hypothetical protein (GeneDB accession Tb927.6.5070, NCBI accession no. XP_826216). The CAD spectrum shown was not of sufficient quality to identify this peptide. For clarity, only singly charged products are labeled, although a number of doubly charged $\mathrm{c}$ and $\mathrm{z}$ series ions were identified. 


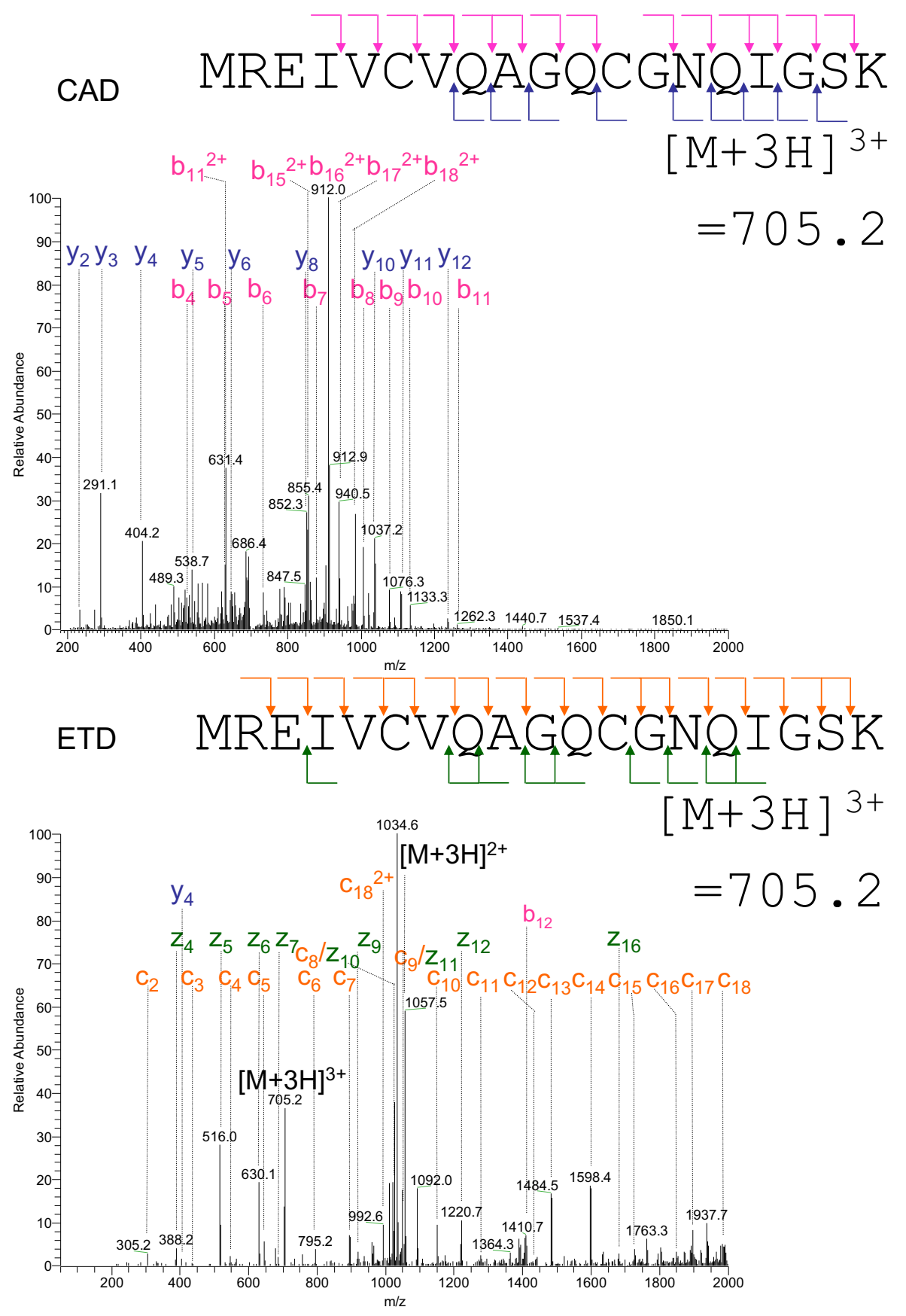

Figure 1. Continued.

specific fragmentations, with a far greater diversity of detectable fragmentation pathways in comparison to CAD. Similar trends in the fragmentation efficiency of multiply charged peptidic precursors by have been reported previously by others $[20,26]$. The net solution-phase charge of the peptides has been investigated and indicates that, as expected, basic peptides are more predominant in the set of peptides identified using ETD than in those identified by CAD (see Supplementary Figure 2).

As a result of the strong qualitative variations in spectroscopic data mentioned above, determining best practice for comparison of the results from database searching of these data types was important; since the product ion spectra types investigated here are so different, this invalidates the use of identical filtering parameters following database searching.

Recognition of Peptides and Proteins Using ETD and CAD of Peptide Ions

As described above, clear qualitative differences were observed between CAD and ETD spectra. We therefore adopted a conservative approach for filtering of the 


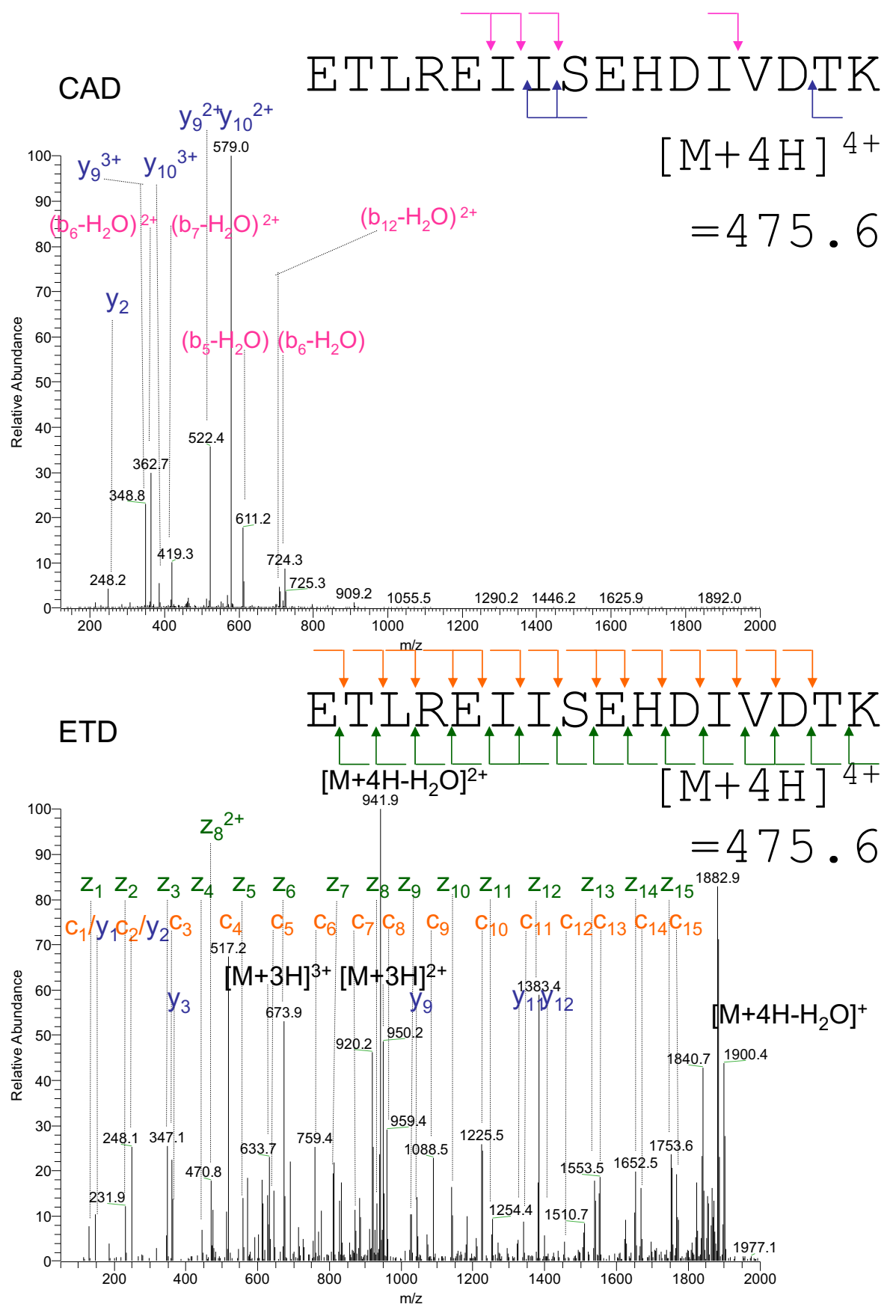

Figure 1. Continued.

resultant identification data. The results of our database searching were normalized with respect to maintaining a set FDR rather than using set filtering parameters. Similarly to Gygi and coworkers' approach, we employed the cross-correlation coefficient, XCorr, which indicates goodness of fit between theoretical and observed spectrum, and the relative difference in score between the top "hit" and next match, $\Delta \mathrm{Cn}$ as filtering parameters for our data [22, 25]. Values of XCorr and $\Delta C n$ generating a $1 \%$ FDR within each charge state were ascertained, and the numbers of peptides matched using these parameters are summarized in Table 1 and Supplementary
Figure 3. The results of the database search, using varying FDR are summarized in Figure 2, which illustrates the number of (nonredundant) peptides matched at a FDR of either $1 \%$ (Figure $2 \mathrm{a}$ ) or $5 \%$ (Figure $2 \mathrm{~b}$ ). The FDR was determined by examination of the number of hits matching to "decoy" sequences within a concatenated forward-reverse database search [20]. A sub-set of the data was subjected to database searching following filtering to remove $\mathrm{m} / \mathrm{z}$ values consistent with the presence of residual and charge-reduced precursor ion species; this additional processing step was found to have no significant influence upon the resultant peptide recognitions made (data not shown). 
Table 1. Sequest search data filtering parameters: values of XCorr and $\Delta C n$ that generated a false discovery rate of $1 \%(5 \%$ in parentheses), and resultant numbers of peptide identifications at charge state $\mathrm{z}$

\begin{tabular}{lclcccc}
\hline$z$ & XCorr CAD & $\Delta$ Cn CAD & No. peptides CAD & XCorr ETD & $\Delta$ Cn ETD & No. peptides ETD \\
\hline \hline 2 & $2.1(1.9)$ & $0.26(0.16)$ & $1938(2544)$ & $1.8(1.6)$ & $0.38(0.30)$ & $152(426)$ \\
3 & $2(2.0)$ & $0.38(0.3)$ & $837(1198)$ & $3.0(2.7)$ & $0.22(0.15)$ & $1649(1944)$ \\
4 & $2.7(2.3)$ & $0.38(0.37)$ & $163(209)$ & $3.6(3.4)$ & $0.23(0.14)$ & $650(711)$ \\
5 & $2.4(2.4)$ & $0.41(0.41)$ & $17(17)$ & $4.3(4.0)$ & $0.08(0.20)$ & $75(82)$ \\
\hline
\end{tabular}

Perl script filtering of concatenated forward-reverse database searching using Sequest to determine the values of $X C o r r$, $\Delta$ Cn, which gave the maximal number of peptide recognitions at a $1 \%$ ( $5 \%$ in brackets) FDR for each charge state (see the Experimental section for details). Numbers of peptides of each charge state at the stated FDR cutoff are as indicated.

Maintaining our peptide identification stringency at $1 \%$ FDR and allowing protein assignment where two or more independent peptide identifications were made using one fragmentation technique alone (i.e., either

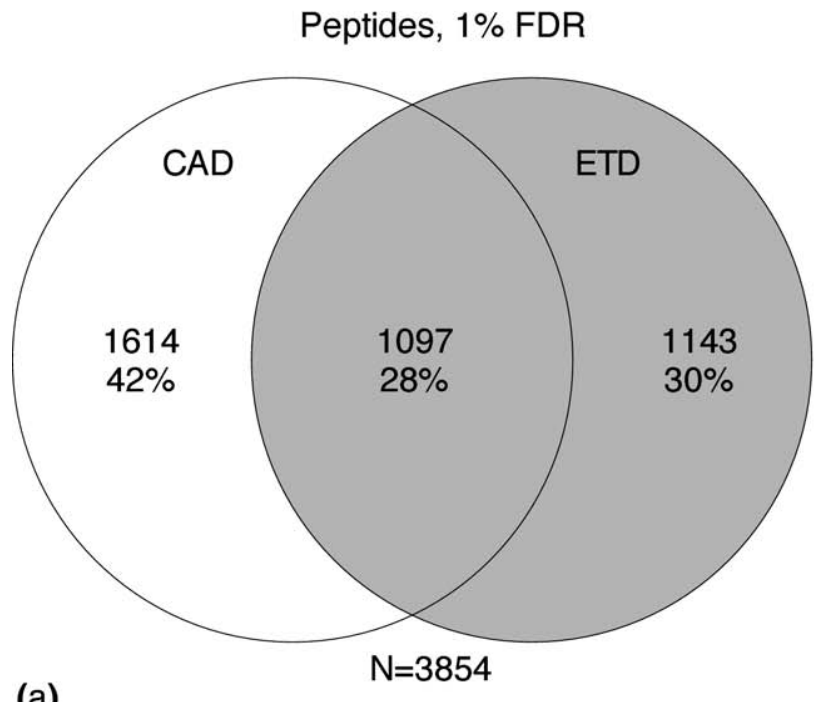

(a)

Peptides, 5\% FDR

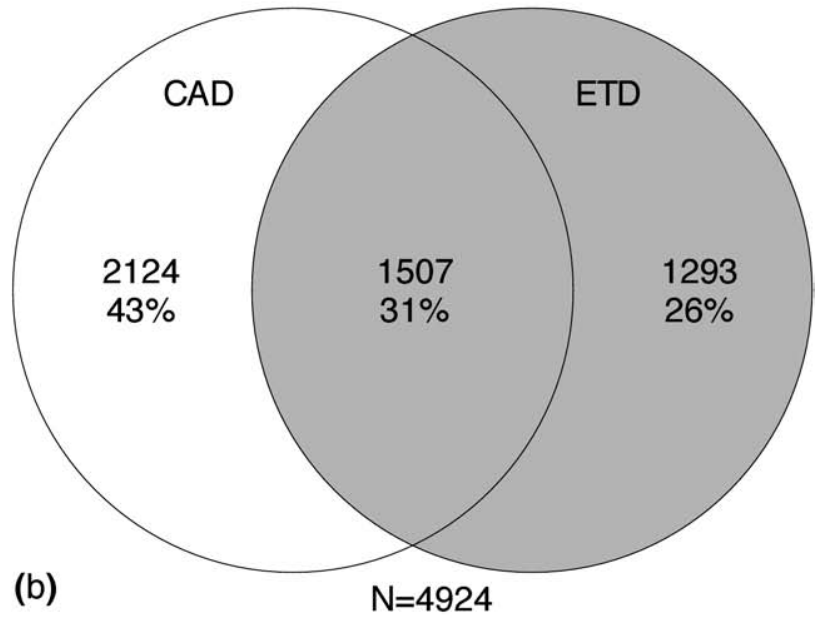

Figure 2. Overlap between peptides identified using dissociation via collisional or electron-transfer activation. Product ion spectra were converted to text-formatted .dta files, sorted according to their origin (CAD or ETD) using a custom-written batch file, and subjected to identification using TurboSequest. Peptide identifications were subjected to (XCorr, $\Delta \mathrm{Cn}$ ) filtering to gain an FDR of $1 \%$ (a) or 5\% (b), see Experimental for details.
CAD or ETD), recognition of protein presence was examined (See Figure 3 and Supplementary Table 1). Slightly more protein recognitions were made on the basis of CAD data alone versus ETD alone (19.8\% versus $13.8 \%$ ), and $66.3 \%$ were recognized independently by both activation methods (CAD and ETD). Hence using both ETD and CAD not only substantiated the assignment of proteins independently recognized by each approach alone, with 264 proteins being recognized by both CAD and ETD, but also noticeably augmented the overall number of protein assignments in comparison to using only ETD or CAD. Since precisely the same peptide precursors were subjected to fragmentation by both ETD and CAD, the variation in peptide and protein identification resulting from these two analytical conditions reflects distinct identifications made from the two fragmentation methods rather than stochastic variation in the peptides observed between one experiment and the next. Similar findings were reported in a recent comparison of the results of electron capture and collisional activation [16].

Proteins, $1 \%$ FDR ( $5 \%$ FDR), $\geq 2$ peptides

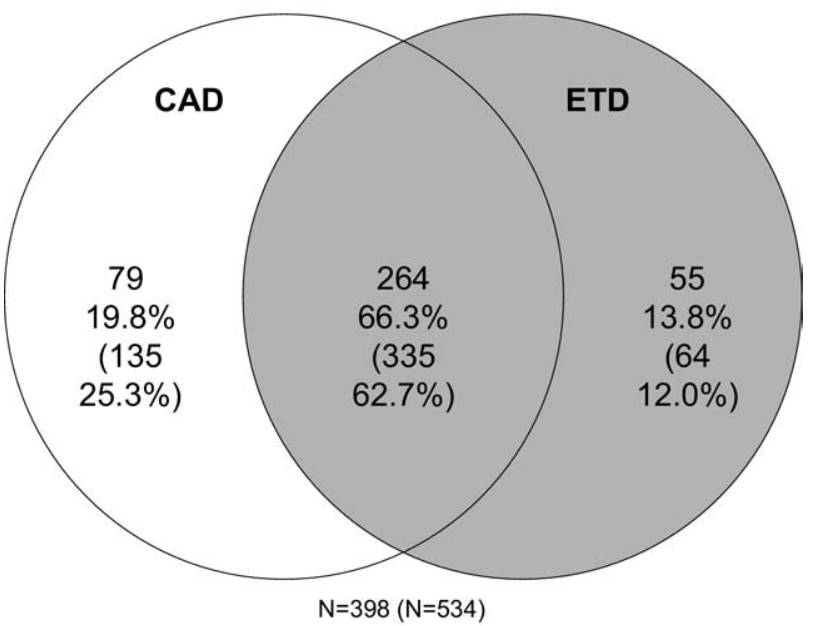

Figure 3. Comparison of the identified proteins from collisional versus electron-transfer-mediated peptide dissociation. Proteins that were confidently recognized using data generated by ETD and/or CAD according to the criteria stated in the main text (1\% FDR, $\geq 2$ independent peptides identified), were output as text files and subjected to comparison using custom-written Perl scripts. Figures in brackets indicate protein recognitions at a relaxed FDR of $5 \%, \geq 2$ independent peptides identified). 
Cases where two individual peptide identifications were made to a putative gene product (one by ETD, one by CAD) were examined (see Supplementary Tables 1 and 2); these data further supplement our argument. A number of additional peptide recognitions improve the sequence coverage if individual peptide recognitions, one arising from each fragmentation method, are considered (see Supplementary Table 1). Indeed, if two individual peptide recognitions are considered, one of each resulting from either CAD or ETD fragmentation of different precursors, an additional 34 protein recognitions can be made, 12 of which were not observed in our previous analysis of the TbFP (see Supplementary Table 2). In a limited number of cases, these peptides show some overlap (i.e., they result from missed enzymatic cleavage sites); these remain independent recognitions of distinct peptide analytes. The findings reported here are similar to those found in a recent paper from Coon and coworkers, which described analyses performed upon Arabidopsis thaliana and Saccharomyces cerevisiae using CAD/ETD switching methods [20]. The reported extent of new peptide recognitions arising from performing ETD analysis, and the extent of overlap between peptide recognitions made via ETD and CAD was substantially lower than that found in this study ( $12 \%$ of peptides, with overall $0.48 \%$ FDR versus $28 \%$ at $1 \%$ FDR in the current analysis). To some extent, this difference may arise from differences in data acquisition, processing and database search methods; for instance, Good et al. consider each charge state of a single peptide as a unique entity, whilst we have considered peptide sequences and removed redundancy attributable to the presence of multiple charge states for an individual peptide from consideration of overlap. The distribution of peptide length for ETDrecognized peptide analytes is skewed somewhat towards longer peptides than that of analytes identified by CAD (mean 15 residues for ETD versus 14 for CAD). Similarly, peptides recognized by ETD are more basic in nature than those recognized by CAD (Supplementary Figures 1 and 2), showing similar trends to the published Arabidopsis and yeast data [20]. A similar strategy employing normalization of FDR for ETD and CAD data was recently reported by Pandey and coworkers [27], in their case employing a different search engine upon a proprietary mixture of 50 proteins.

Direct comparison with our previous analysis of the TbFP [21] is complicated by a number of experimental and informatic factors. Different sample preparation, analysis, database searching, and data filtering parameters have been used in both cases, and the resultant sets of identified proteins therefore show numerous differences. To some degree, this variance is attributable to routine undersampling, akin to that observed in all proteomic experiments upon relatively complex samples. In addition, in this case, since no biological assumptions are applied to our data, pI-based filtering of putative identifications has been applied only to this dataset post hoc; indeed, our previous analyses con- firmed that some bona fide flagellar proteins are removed by this filtering [21]. All data are presented in Supplementary Table 1 . In spite of these analytical differences, an important finding is that a substantial number of proteins were found in all three proteome investigations (the previously-published TbFP, the CAD, and ETD data presented here) -238 proteins in all. Confidence in the recognition of this "core set" of proteins is heightened because two entirely independent datasets (and furthermore two independent activation methods within the current experiment) have agreed in recognizing the presence of these proteins. One hundred sixty-eight proteins that were previously not observed have been recognized within the ETD/ CAD data generated in this study. As with any proteome analysis-based prediction of gene function, the confirmation of these proteins as bona fide flagellar proteins requires further experimental validation; however we do note that a number of previously characterized flagellar proteins absent from the published TbFP are present in this new proteomic dataset.

\section{Conclusions}

The results of the experiments described here and of the analyses performed upon the resultant mass spectrometric data indicate that a combined ETD/CAD approach is far superior to applying either approach alone. Using this switching method, approximately two-thirds of protein assignments made resulted from multiple peptide identifications performed independently by both fragmentation techniques (ETD and CAD). It is important to note that inclusion of increasingly tentative protein assignments (such as those suggested by application of filtering parameters that result in a higher FDR) may in general be justified if they are used to prompt appropriately targeted biological experiments (e.g., knockdown and immunofluorescence measurements) to confirm subcellular localization and protein function, or other means of substantiating the assignments thus generated.

Due to the strong differences between both the precursors, which generate optimal data using each activation method and the nature of the product ion spectra obtained, it is critical that validation of data obtained using these CAD and ETD approaches is performed using an objectively stringent method. We therefore advocate that the most appropriate method for performing this comparison is to filter data using a set FDR, allowing different filtering parameters to be applied.

\section{Acknowledgments}

The authors acknowledge support provided by the BBSRC Exploiting Genomics program (BBSRC grants EGH17742, EGH17741, EGM17685, and EGH17729), for SRH, RB, KWL, and NP, respectively). SRH and KWL are currently supported by the EPSRC 
(grants EP/E043143/1 and EP/D013615/1, respectively). The authors thank the reviewers for helpful suggestions.

\section{References}

1. Simpson, R. J.; Connolly, L. M.; Eddes, J. S.; Pereira, J. J.; Moritz, R. L.; Reid, G. E. Proteomic Analysis of the Human Colon Carcinoma Cell Line (LIM 1215): Development of a Membrane Protein Database. Electrophoresis 2000, 21, 1707-1732.

2. Steen, H.; Mann, M. The ABC's (and XYZ's) of Peptide Sequencing. Nat. Rev. Mol. Cell Biol. 2004, 5, 699-711.

3. Aebersold, R.; Goodlett, D. R. Mass Spectrometry in Proteomics. Chem. Rev. 2001, 101, 269-295.

4. Larsen, M. R.; Trelle, M. B.; Thingholm, T. E.; Jensen, O. N. Analysis of Post-Translational Modifications of Proteins by Tandem Mass Spectrometry. Biotechniques 2006, 40, 790-798

5. Smith, R. D.; Barinaga, C. J.; Udseth, H. R. Tandem Mass-Spectrometry of Highly Charged Cytochrome $c$ Molecular-Ions Produced by Electrospray Ionization. J. Phys. Chem. 1989, 93, 5019-5022

6. Nemeth-Cawley, J. F.; Rouse, J. C. Identification and Sequencing Analysis of Intact Proteins Via Collision-Induced Dissociation and Quadrupole Time-of-Flight Mass Spectrometry. J. Mass Spectrom. 2002, 37, $270-282$

7. Raspopov, S. A.; El-Faramawy, A.; Thomson, B. A.; Siu, K. W. Infrared Multiphoton Dissociation in Quadrupole Time-of-Flight Mass Spectrometry: Top-Down Characterization of Proteins. Anal. Chem. 2006, 78, 4572-4577.

8. Zubarev, R. A.; Kelleher, N. L.; McLafferty, F. W. Electron Capture Dissociation of Multiply Charged Protein Cations. A Nonergodic Process. J. Am. Chem. Soc. 1998, 120, 3265-3266.

9. Zubarev, R. A.; Horn, D. M.; Fridriksson, E. K.; Kelleher, N. L.; Kruger, N. A.; Lewis, M. A.; Carpenter, B. K.; McLafferty, F. W. Electron Capture Dissociation for Structural Characterization of Multiply Charged Protein Cations. Anal. Chem. 2000, 72, 563-573.

10. Breuker, K.; Oh, H.; Lin, C.; Carpenter, B. K.; McLafferty, F. W. Nonergodic and Conformational Control of the Electron Capture Dissociation of Protein Cations. Proc. Natl. Acad. Sci. U.S.A. 2004, 101, 14011-14016.

11. Syrstad, E. A.; Turecek, F. Toward a General Mechanism of Electron Capture Dissociation. J. Am. Soc. Mass Spectrom. 2005, 16, 208-224.

12. Hudgins, R. R.; Håkansson, K.; Quinn, J. P.; Hendrickson, C. L.; Marshall, A. G., 50th American Society for Mass Spectrometry Conference on Mass Spectrometry and Allied Topics, May 2002, Orlando, FL.

13. Zubarev, R. A. Reactions of Polypeptide Ions with Electrons in the Gas Phase. Mass Spectrom. Rev. 2003, 22, 57-77.
14. Chalmers, M. J.; Hakansson, K.; Johnson, R.; Smith, R.; Shen, J.; Emmett, M. R.; Marshall, A. G. Protein Kinase A Phosphorylation Characterized by Tandem Fourier Transform Ion Cyclotron Resonance Mass Spectrometry. Proteomics 2004, 4, 970-981.

15. Cooper, H. J.; Hakansson, K.; Marshall, A. G. The Role of Electron Capture Dissociation in Biomolecular Analysis. Mass Spectrom. Rev. 2005, 24, 201-222.

16. Creese, A. J.; Cooper, H. J. Liquid Chromatography Electron Capture Dissociation Tandem Mass Spectrometry (LC-ECD-MS/MS) Versus Liquid Chromatography Collision-Induced Dissociation Tandem Mass Spectrometry (LC-CID-MS/MS) for the Identification of Proteins. J. Am. Soc. Mass Spectrom. 2007, 18, 891-897.

17. Syka, J. E.; Coon, J. J.; Schroeder, M. J.; Shabanowitz, J.; Hunt, D. F. Peptide and Protein Sequence Analysis by Electron Transfer Dissociation Mass Spectrometry. Proc. Natl. Acad. Sci. U.S.A. 2004, 101, 9528 9533.

18. Brekenfeld, A.; Ledertheil, T.; Lubeck, M.; Baessmann, C.; Hartmer, R., 53rd American Society for Mass Spectrometry Conference on Mass Spectrometry and Allied Topics, May 2005, San Antonio, TX.

19. Coon, J. J.; Ueberheide, B.; Syka, J. E.; Dryhurst, D. D.; Ausio, J. Shabanowitz, J. Hunt, D. F. Protein Identification Using Sequential Ion/Ion Reactions and Tandem Mass Spectrometry. Proc. Natl. Acad. Sci. U.S.A. 2005, 102, 9463-9468.

20. Good, D. M.; Wirtala, M.; McAlister, G. C.; Coon, J. J. Performance Characteristics of Electron Transfer Dissociation Mass Spectrometry. Mol. Cell. Proteom. 2007, 6, 1942-1951.

21. Broadhead, R.; Dawe, H. R.; Farr, H.; Griffiths, S.; Hart, S. R.; Portman N.; Shaw, M. K.; Ginger, M. L.; Gaskell, S. J.; McKean, P. G.; Gull, K. Flagellar Motility is Required for the Viability of the Bloodstream Trypanosome. Nature 2006, 440, 224-227.

22. Peng, J.; Elias, J. E.; Thoreen, C. C.; Licklider, L. J.; Gygi, S. P. Evaluation of Multidimensional Chromatography Coupled with Tandem Mass Spectrometry (LC/LC-MS/MS) for Large-Scale Protein Analysis: The Yeast Proteome. J. Proteome Res. 2003, 2, 43-50.

23. Lundgren, D. H.; Han, D. K.; Eng, J. K. Protein Identification using TurboSEQUEST. Curr. Protoc. Bioinformatics 2005, Chap. 13, Unit 13.3.

24. Good, D. M.; Coon, J. J. Advancing Proteomics with ion/ion Chemistry. Biotechniques 2006, 40, 783-789.

25. Elias, J. E.; Gygi, S. P. Target-Decoy Search Strategy for Increased Confidence in Large-Scale Protein Identifications by Mass Spectrometry. Nat. Methods 2007, 4, 207-214.

26. Swaney, D. L.; McAlister, G. C.; Wirtala, M. Schwartz, J. C.; Syka, J. E. Coon, J. J. Supplemental Activation Method for High-Efficiency ElectronTransfer Dissociation of Doubly Protonated Peptide Precursors. Anal. Chem. 2007, 79, 477-485.

27. Molina, H.; Matthiesen, R.; Kandasamy, K.; Pandey, A. Comprehensive Comparison of Collision Induced Dissociation and Electron Transfer Dissociation. Anal. Chem. 2008, 80, 4825-4835. 